\title{
NMR on Surfaces: Present Achievements and Future Prospects*
}

\author{
D. FICK $^{\dagger}$ \\ Philipps-Universität, Fachbereich Physik and Center for Material Science \\ 35032 Marburg, Germany \\ $\beta$-NMR experiments with hyperpolarized ${ }^{8} \mathrm{Li}$ adsorbed on semiconduc- \\ tor and metal surfaces are described (adsorption on $\mathrm{Si}(111)-(7 \times 7), \mathrm{Si}(111)-\mathrm{H}$, \\ $\mathrm{Si}(111)-(1 \times 1): H, \operatorname{Ru}(001))$. They yield detailed information on the electronic \\ properties of these adsorbate-surface systems. Briefly a scenario using hy- \\ perpolarized Xe isotopes is sketched, which will extend the potential of the \\ use of hyperpolarized species in NMR experiments on surfaces.
}

PACS numbers: $68.35 .-\mathrm{p}, 68.47 . \mathrm{De}$

\section{Introduction}

Nuclear magnetic resonance (NMR) is one of the most powerful tools to study liquids and solids, since direct information can be obtained on the chemical environment, on the local geometry and very important on dynamical processes, as electron fluctuations, diffusion, hopping etc. Its applications range from solid-state physics to analytical chemistry, protein structure investigation, and medical NMR imaging. Despite this large potential, very little of the NMR techniques have been applied to study properties of single crystal surfaces, since their use is hampered by the inherently low sensitivity of NMR. In a typical solid-state application a minimum number of $10^{17}$ to $10^{19}$ equivalent nuclei must be present in the probe [1]. With approximately $10^{15}$ adsorption sites on $1 \mathrm{~cm}^{2}$ surface area of a typical laboratory single crystal, there is a discrepancy of more than two orders of magnitude in sensitivity. It increases still further if adsorbate properties at low coverages are to be investigated. This might happen e.g. in diffusion studies if mutual interaction of the adsorbates ought to be avoided.

\footnotetext{
*Supported partly by the Bundesministerium für Bildung und Forschung and the Deutsche Forschungsgemeinschaft, Bonn.

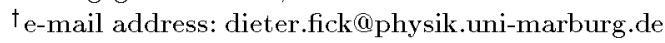


The use of "hyperpolarized" adsorbates circumvent these difficulties. In particular, for the alkali-metal adsorbates over the last decade special methods have been developed to overcome the sensitivity problem on single crystal surfaces. They all use highly nuclear spin polarized ("hyperpolarized") atomic beams. Utilizing the stable isotopes as ${ }^{6,7} \mathrm{Li}$ and ${ }^{23} \mathrm{Na}[2,3]$ the experimental techniques were deduced from techniques originally developed for nuclear reaction experiments with polarized heavy ions [4]. Certainly, in particular the use of the "small" Li atom requires careful checks whether it really adsorbs on the surface, or penetrates into the bulk $[5,6]$.

We will report here mainly on present achievements on $\beta$-NMR experiments with hyperpolarized ${ }^{8} \mathrm{Li}$ atomic beams for which a UHV suitable source has been built at the Heidelberg MP tandem accelerator [7]. Experimental results for the uncovered and hydrogen covered $\operatorname{Si}(111)-(7 \times 7)$ and the $\mathrm{Ru}(001)$ surfaces dealing mainly with the electronic structure and with phase transitions will be discussed briefly. Finally, a new development using adsorption of hyperpolarized ${ }^{129} \mathrm{Xe}$ gas in connection with a conventional NMR spectrometer, to which a UHV chamber had been added, will be presented.

\section{Experimental}

The idea to use $\beta$-(detected) NMR with highly polarized ${ }^{8} \mathrm{Li}$ adsorbates to overcome the NMR sensitivity problem stems from the experience in solid-state $\beta$-NMR experiments [8, 9]. However, opposite to such experiments in which polarized recoiled nuclei from nuclear reactions can be used, $\beta$-NMR on surfaces requires a gentle landing of the adsorbates and thus thermal nuclear spin polarized ${ }^{8} \mathrm{Li}$ atoms (nuclear spin $I=2$ ). Because of the half life of ${ }^{8} \mathrm{Li}$ of about $0.8 \mathrm{~s}$ the experiment has to be situated at an accelerator, here the MP-Tandem accelerator in Heidelberg.

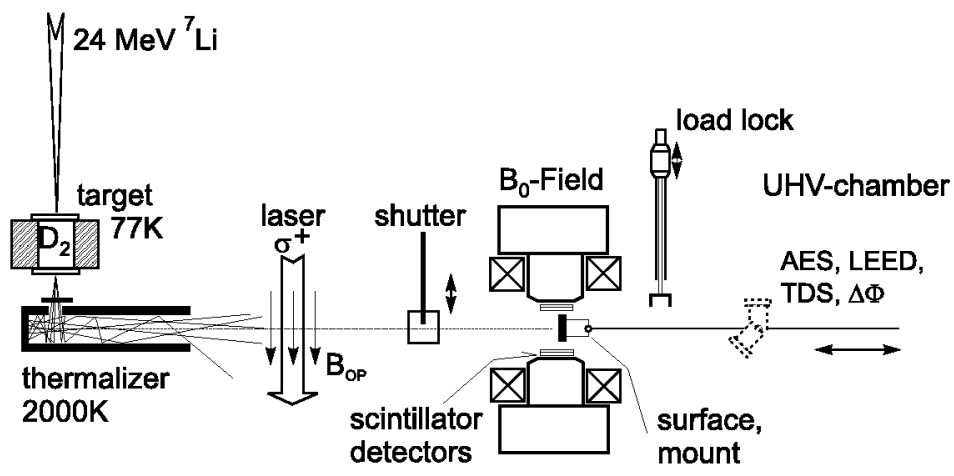

Fig. 1. Schematic view of the experimental setup. A description is given in the text. 
A sketch of the most important components of the experimental setup is given in Fig. 1. It consists of four essential parts: a bakeable source for a thermal nuclear spin polarized ${ }^{8} \mathrm{Li}$ beam, an NMR region including detectors for the ${ }^{8} \mathrm{Li}$ decay electrons, a fast load lock, and a conventional UHV chamber with standard analysis tools. Most of the equipment has been described in some detail recently $[7,10-12]$. The ${ }^{8} \mathrm{Li}$ isotope is produced in the ${ }^{2} \mathrm{H}\left({ }^{7} \mathrm{Li},{ }^{8} \mathrm{Li}\right) \mathrm{p}$ reaction from a $24 \mathrm{MeV}{ }^{7} \mathrm{Li}^{3+}$ ion beam. To ensure the gentle "landing" on the surfaces and not an implantation of the ${ }^{8} \mathrm{Li}$ the fast reaction products are thermalized by implanting them into a tubular graphite stopper, from which they evaporate thermally [7]. The thermal atomic beam thus formed is highly polarized by laser optical pumping. A shutter ensures controlled timing of the atomic beam.

A differential pumping section separates the beam production chamber from the NMR surfaces analysis chamber situated in a magnetic field of up to $0.9 \mathrm{~T}$ provided by an electromagnet. In between pole shoes and the chamber, pairs of plastic scintillator detectors are placed on either side. They are connected to light guides and photomultipliers to detect the decay electrons. Details of the signal detection can be found in Refs. [11, 12].

The crystal can be positioned into the center of the magnet or in front of several analytical tools by a manipulator that also allows for heating and cooling (90 K up to $1500 \mathrm{~K}$ ). The UHV chamber itself has a base pressure of $5 \times 10^{-11} \mathrm{mbar}$. The crystals are cleaned using conventional methods as argon ion bombardment and prolonged heating, sometimes in an oxygen atmosphere, followed by a sequence of flashes and annealing steps. Additional lithium can be dosed onto the surface using thoroughly outgased SAES dispenser sources.

In between the UHV chamber and the NMR region a home-made load lock is installed, built mainly for a fast transfer of wet chemically prepared hydrogen terminated $\mathrm{Si}(111)-(1 \times 1)$ :H surfaces [13]. Most of the construction is made from non-magnetic steel to avoid any kind of stray magnetic fields during NMR experiments. From feeding the load lock with the sample it lasts about four minutes to reach $5 \times 10^{-8}$ mbar and after opening the valve to the UHV chamber another seven minutes until the sample is in an UHV environment of $3 \times 10^{-11} \mathrm{mbar}$.

${ }^{8} \mathrm{Li}$ is a $\beta$-decaying nucleus with a half-life of $T_{1 / 2}=0.84 \mathrm{~s}$. Spin polarization of the adsorbate ${ }^{8} \mathrm{Li}$ itself can therefore be detected via the directional asymmetry of the $\beta$-decay electron (end-point energy $12.4 \mathrm{MeV}$ ). The nuclear polarization $P$ of the ${ }^{8} \mathrm{Li}$ ensemble is determined from the observed asymmetry $\epsilon$ of the $\beta$-electron intensity with respect to the magnetic field as

$$
\epsilon=\frac{N\left(0^{\circ}\right)-N\left(180^{\circ}\right)}{N\left(0^{\circ}\right)+N\left(180^{\circ}\right)}=-\frac{1}{3} P .
$$

Here $N\left(0^{\circ}\right)$ denotes the count rates for electrons emitted along the direction of the magnetic field, while $N\left(180^{\circ}\right)$ denotes the count rate for electrons emitted opposite to it. The factor $\left(-\frac{1}{3}\right)$ is due to the nuclear properties of the ${ }^{8} \mathrm{Li} \beta$-decay. Systematic 
errors in the determination of $\epsilon$ are eliminated by performing the experiment with the reversed polarization $P$ as well.

The nuclear spin relaxation experiments consist basically of three subsequent steps which are repeated many times. It starts with a collecting period (typically $1.5 \mathrm{~s}$ ) during which ${ }^{8} \mathrm{Li}$ is produced and accumulated in the thermalizer (Fig. 1). Afterwards it is released while production is still going on. An atomic beam is formed and nuclear spin polarized by optical pumping. The thermal ${ }^{8} \mathrm{Li}$ atoms are accumulated on the surface for $0.5 \mathrm{~s}$. Finally, during the detection period of $4 \mathrm{~s}$ the $\beta$-electron asymmetry $\epsilon$ (Eq. (1)) is measured as a function of time. During the detection period the ion beam, as the main source of background signals, is switched off. Meanwhile, it is also possible to measure the asymmetry during the activation period taking properly into account the large background caused by neutrons which are generated by nuclear reactions initiated by the $24 \mathrm{MeV}{ }^{7} \mathrm{Li}$ beam.

The observed exponential time dependence of $\epsilon(t)$ or $P(t)$, Eq. (1), bears the information on the nuclear spin relaxation rate $\alpha$ or its inverse, the $T_{1}$ time, well known from NMR literature [14-16]:

$$
\epsilon(t)=\epsilon(0) \mathrm{e}^{-\alpha t}=\epsilon(0) \mathrm{e}^{-t / T_{1}} .
$$

Contrary to conventional NMR experiments the determination of the $T_{1}$ time does not require the application of resonant rf fields, since the nuclear spin polarization $P$ in the thermal equilibrium (of the order of $10^{-6}$ ) can be neglected compared to the initial polarization of the ${ }^{8} \mathrm{Li}$ ensemble of 0.8 to 0.9 after adsorption.

\section{The $\operatorname{Si}(111)-(7 \times 7)$ surface}

\subsection{The metallicity of the $(7 \times 7)$ reconstruction}

Due to its dangling bonds the $(7 \times 7)$ reconstruction of the $\operatorname{Si}(111)$ surface has around room temperature many hall marks of a metallic surface. Recently, not only a parabolic dispersive band located in the center of the $(7 \times 7)$ Brillouin zone has been identified [17], but also "Korringa-like nuclear spin relaxation" was observed in NMR experiments on Li, adsorbed at extremely low coverages of $10^{-4}$ monolayer (ML) and below [18]. Relying on common wisdom, the observation of "Korringa-like nuclear spin relaxation" is considered as a univocal sign for the "metallicity" of a system [14-16, 19]. In what follows, we will be more careful and consider it as a sign for an at least partly delocalized electron gas with correlation times much smaller than the inverse electron Larmor frequency at the magnetic field used. It derives mainly from adatom dangling bonds which are only partially occupied [20,21]. Since it is known, at least theoretically, that Li adsorbs mainly at these adatoms [22], we expect that nuclear spin relaxation experiments on Li adsorbed with very small coverage probe the electron correlations generating the dispersive band. 
The previous experiments [18] were meanwhile extended to higher magnetic fields and performed under much better vacuum conditions using the newly installed UHV suitable polarized ${ }^{8} \mathrm{Li}$ source described in Sec. 2. While taking $T_{1}$ data on Li adsorbed on the clean $\mathrm{Si}(111)-(7 \times 7)$ surface it was flashed each 10 min to about $500 \mathrm{~K}$ and each one to two hours to $1000 \mathrm{~K}$, thus preventing non-polarized ${ }^{7} \mathrm{Li}$ which represents the major part of the atomic beam to accumulate to a considerable fraction on the surface. In such a way at most $10^{-4} \mathrm{ML}$ of $\mathrm{Li}$ was accumulated (that is one Li atom per 200 unit cells of the $(7 \times 7)$ reconstruction).

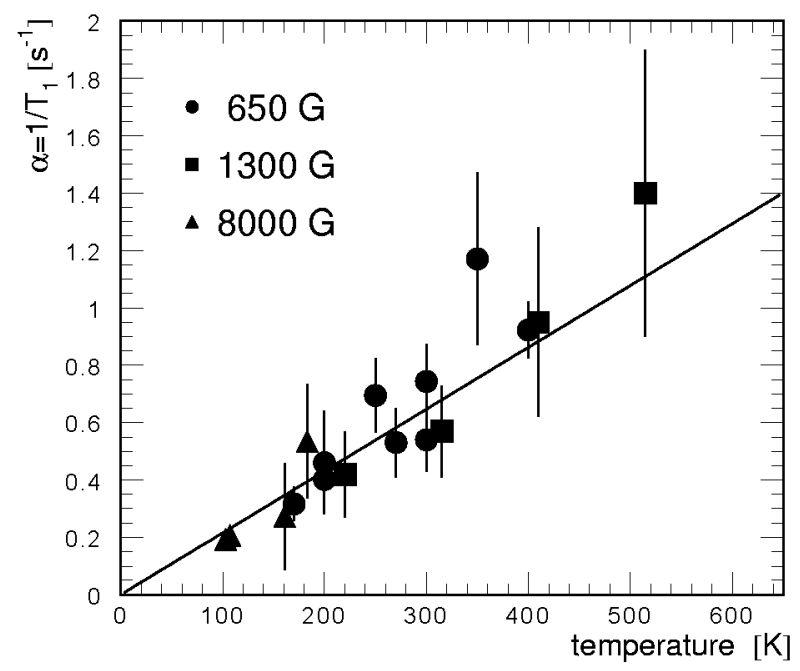

Fig. 2. Nuclear spin relaxation rates $\alpha=1 / T_{1}$ as a function of substrate temperature for three different magnetic fields for ${ }^{8} \mathrm{Li}$ adsorbed on a clean $\operatorname{Si}(111)-(7 \times 7)$ surface. Data were observed for $n$ - and $p$-doped material, respectively.

Figure 2 displays the data for $\alpha=1 / T_{1}$ as a function of temperature [13]. They are independent of magnetic field strength and independent of doping, as shown already previously [18]. Doubtless, the data follow quite well a linear temperature dependence which can be characterized by $T_{1} T=(498 \pm 117) \mathrm{s} \mathrm{K}$. This value is in perfect agreement with the slope of previous data [18]. Literally, the properties of these data may therefore be taken as a sign of the metallicity of the $(7 \times 7)$ reconstruction of the $\mathrm{Si}(111)$ surface. However, since the relaxation rates $\alpha=1 / T_{1}$ exceed by about $70 \%$ those found under similar circumstances for ${ }^{8} \mathrm{Li}$ adsorbed on the metal surface $\operatorname{Ru}(001)\left(T_{1} T=(852 \pm 131) \mathrm{s} \mathrm{K},[23]\right.$ and Sec. 5) a more detailed discussion is indicated.

And indeed, for the "metallic" $(7 \times 7)$-reconstruction, we cannot expect that like for "real" metals the description of nuclear spin relaxation rates can be based on "free" electrons, occupying plane wave Bloch states (non-interacting electron gas). As well the recently found narrow band around $\epsilon_{\mathrm{F}}[17]$ as the already men- 
tioned larger relaxation rates as compared to those of ${ }^{8} \mathrm{Li}$ adsorbed on the $\mathrm{Ru}(001)$ metal surface point to an increased electron localization. Here, it is worthwhile to mention additionally that on the basis of a Hubbard-like Hamiltonian describing the degenerate electron gas of the adatom dangling bonds, hopping integrals of $25 \mathrm{meV}$ and $75 \mathrm{meV}$ were found leading to a band around the Fermi energy with a width of only $100 \mathrm{meV}[20,21]$. This value is considerably smaller than the band width of $300 \mathrm{meV}$ found experimentally [17]. Certainly, it is a matter of taste, whether one wants to call such a system still metallic or not. But the correlation times of a few $10^{-14} \mathrm{~s}$ associated to such a picture are still short enough that "Korringa relaxation" would be visible (see below).

For such complex system as low coverage Li adsorption on $\mathrm{Si}(111)-(7 \times 7)$ $T_{1}$ times are at present not predictable theoretically even using highly parallelized codes. To obtain nevertheless at least a qualitative picture of what is going on we follow here arguments originally developed for similar experiments in liquid semiconductors [24] and use the correlation time formalism to derive an approximate analog of the standard expression of relaxation rates for a free electron gas. The necessary mutual spin flip of the nucleus and of the electron can only occur for electrons around Fermi energy which yields the linearity of the relaxation rate with surface temperature $T[14-16,19]$. Choosing as correlation times $\tau_{\mathrm{e}}$, the "lifetime of residence" of an electron at the ${ }^{8} \mathrm{Li}$ site (dangling bond), the $T_{1}$ time can be expressed within a factor $\pi / 4$ by the familiar form (Eqs. (14), (21), (22) of Ref. [24])

$$
\alpha=\frac{1}{T_{1}}=\frac{256 \pi^{3}}{9 \hbar} \mu_{\mathrm{e}}^{2}\left(\frac{\mu\left({ }^{8} \mathrm{Li}\right)}{I}\right)^{2}\left|\left\langle|\psi(0)|^{2}\right\rangle\right|_{\mathrm{F}}^{2} \operatorname{DOS}\left(\epsilon_{\mathrm{F}}\right) \tau_{\mathrm{e}} \frac{k T}{\hbar}
$$

$\left(\mu_{\mathrm{e}}, \mu\left({ }^{8} \mathrm{Li}\right)\right.$ magnetic moments, $\left|\left\langle|\Psi(0)|^{2}\right\rangle\right|_{\mathrm{F}}$ probability to find an electron at the nucleus and $\operatorname{DOS}\left(\epsilon_{\mathrm{F}}\right)$ density of states at the Fermi energy). Thus, an increasing correlation time $\tau_{e}$, equivalent to an increasing localization, leads to an enhanced nuclear spin relaxation. This is the basic reason why the relaxation rates for $\mathrm{Li}$ adsorbed on $\mathrm{Si}(111)-(7 \times 7)$ exceeds considerably the one observed for Li adsorption on the metallic $\mathrm{Ru}(001)$ surface.

\subsection{Coverage induced metal-isolator-metal transition}

Photon electron spectroscopy on an alkali-metal $(\mathrm{K})$ covered $\mathrm{Si}(111)-(7 \times 7)$ surface displays with increasing coverage vanishing emission from states near the Fermi energy [25]. That is commonly interpreted as transition from a metallic to a semiconducting surface. A similar behaviour is observed for Li adsorption, but only if the experiments are performed at low temperatures, around $100 \mathrm{~K}$ [26]. It was therefore an obvious idea to determine at that temperature $T_{1}$ times for $\mathrm{Li}$ as a function of coverage.

The coverage dependence of $\alpha=1 / T_{1}$ has been measured at a fixed surface temperature $(110 \mathrm{~K})$ and high magnetic field $(0.8 \mathrm{~T})$ in order to suppress diffusional 


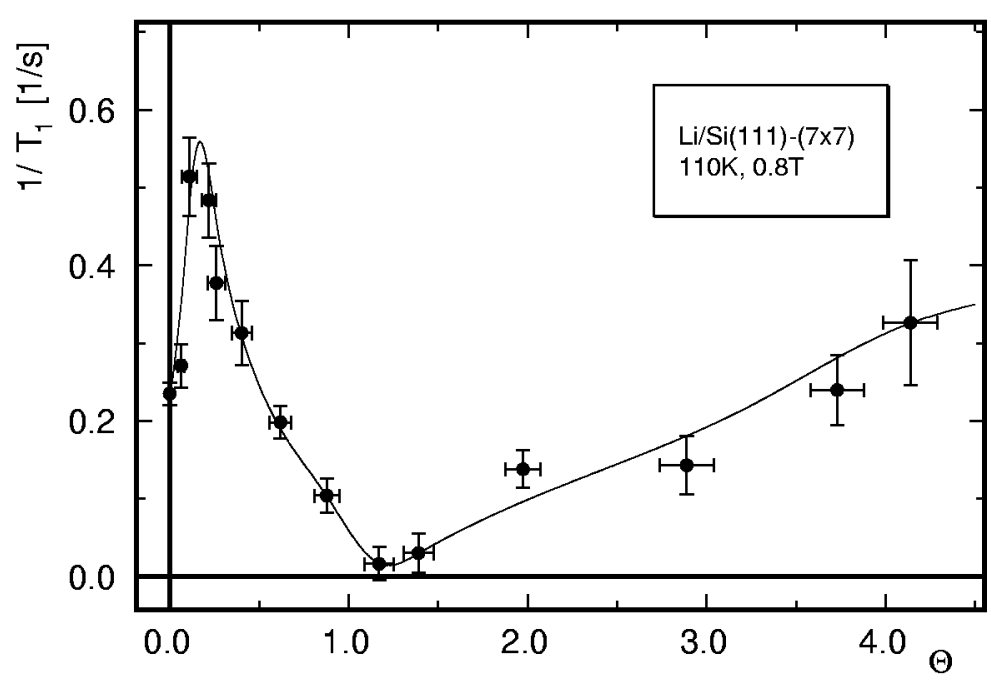

Fig. 3. At a surface temperature of $110 \mathrm{~K}$ and a magnetic field of $0.8 \mathrm{~T}$ nuclear spin relaxation rates as a function of Li-coverage $\Theta$.

relaxation [23]. For each data point the surface has been freshly covered by Li from a getter source. The relaxation rates were then observed for ${ }^{8} \mathrm{Li}$ atoms adsorbed at a very low additional coverage of about $10^{-4}$. Figure 3 displays the data up to a coverage $\Theta$ of about $4 \mathrm{ML}$. (Coverage $\Theta=1$ is equal to one adsorbed $\mathrm{Li}$ atom per Si atom of the $\mathrm{Si}(111)$ surface.) Starting at extremely low coverages the relaxation rate firstly increases with coverage to more than twice its value. Around $\Theta \approx 0.15$ it passes a maximum. At about this coverage all empty dangling bonds of the adatoms are occupied by one unpaired electron: Altogether for the uncovered surface there are 12 adatoms in the $(7 \times 7)$ unit cell, out of which 5 are populated by an electron. They cause the metallicity of the $(7 \times 7)$ reconstruction [20, 21]. As mentioned already above, Li adsorbs preferentially at the adatom dangling bonds [22] filling them further. Obviously at a coverage of $((12-5) / 49 \approx 0.15)$ all dangling bonds are filled with one unpaired electron. This leads to a maximum of the $\operatorname{DOS}\left(\epsilon_{\mathrm{F}}\right)$ (metallicity) and thus to a maximum of the relaxation rate.

Beyond that coverage, dangling bonds are filled further with an additional electron. The surface reconstructs back to a " $1 \times 1$ " like structure [26]. At monolayer coverage all its dangling bonds are filled with electron pairs. They hybridize in a binding and antibinding state. The binding state moves well below Fermi energy generating an isolator (semiconductor) with vanishing $\operatorname{DOS}\left(\epsilon_{\mathrm{F}}\right)$ [25]. Beyond a coverage of $1 \mathrm{ML}$ the relaxation rates increase again and reach at $4 \mathrm{ML}$ a value comparable with the value found for ${ }^{8} \mathrm{Li}$ relaxation in Li metal (at $110 \mathrm{~K}, \alpha=$ $\left.1 / T_{1}=0.35 \mathrm{~s}^{-1}[27]\right)$. The Li overlayer is then fully metallic again and from extremely low coverage to high ones metal-isolator-metal transitions exist on the $\operatorname{Si}(111)$ surface. 


\section{The hydrogen covered and terminated $\mathrm{Si}(111)$ surfaces}

In order to study nuclear spin relaxation of Li adsorbed on a "real" semiconductor surface, temperature dependent relaxation rates $\alpha=1 / T_{1}$ have been measured for Li adsorbed on "cold" and "hot" hydrogen covered, as well as "wet" chemically hydrogen terminated Si(111) surfaces [13] (Fig. 4). As samples modestly $n$ - and $p$-doped crystals with resistivities between 1 and $100 \Omega \mathrm{cm}$ were used. The $\mathrm{Si}(111): H$ samples were prepared by two in situ techniques, both adsorbing atomic $\mathrm{H}$, either onto a "cold" $[28,29]$ or "hot" $[30,31] \mathrm{Si}(111)-(7 \times 7)$ hydrogen covered surface and $\mathrm{Si}(111)-(1 \times 1): \mathrm{H}$ ones by Chabal's ex situ "wet" chemical technique of hydrogen termination [32]. Only the "wet" chemically terminated surfaces have been annealed to about $500 \mathrm{~K}$ for several minutes after the transfer into the UHV.

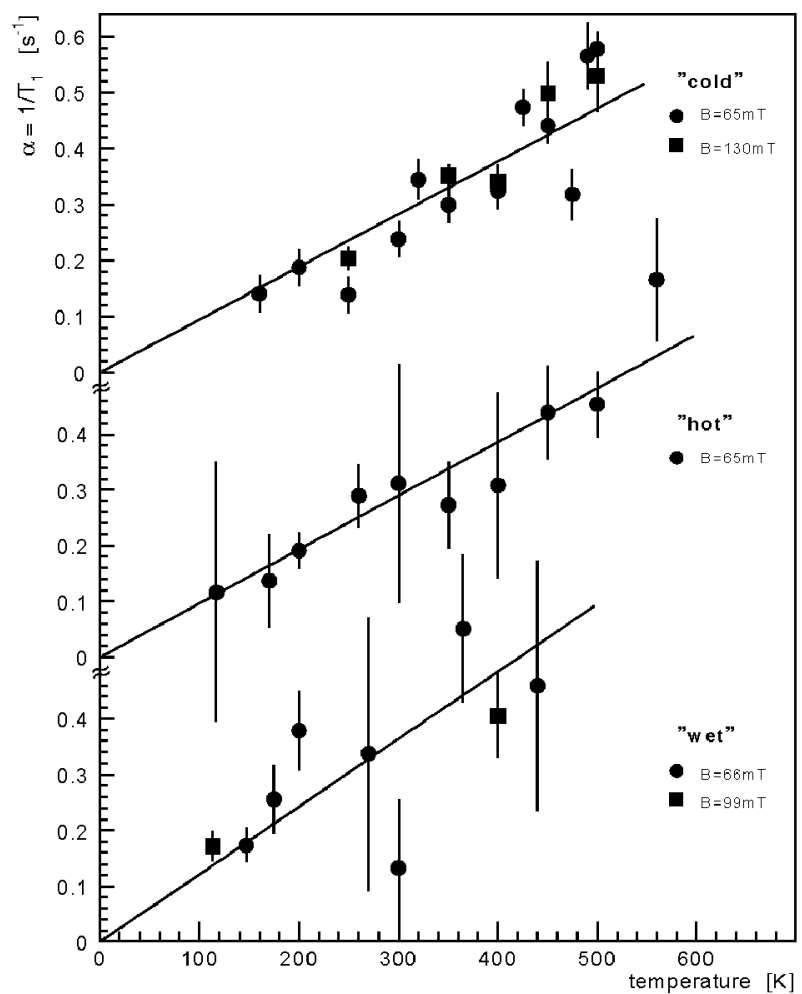

Fig. 4. From above to below nuclear spin relaxation rates as functions of substrate temperature for Li adsorbed on "cold" and "hot" hydrogen covered, as well as "wet" chemical hydrogen terminated $\mathrm{Si}(111)$ surfaces. Various symbols indicate various magnetic fields used. The data are independent of the use of $n$ - or $p$-doped material, respectively. Since the surface could not be flashed each ten minutes, the Li coverage was in between $10^{-3}$ to $10^{-2} \mathrm{ML}$. 
The major difference to the previous measurements on the clean $(7 \times 7)$ reconstruction is that the hydrogen covered and terminated surfaces could not be flashed about every hour to prevent accumulation of non-polarized ${ }^{7} \mathrm{Li}$. Otherwise, the adsorbed hydrogen would have desorbed as well. The data points of Fig. 4 have therefore been obtained by accumulating data for about a day thus accumulating non-polarized ${ }^{7} \mathrm{Li}$ of about $10^{-3}$ to $10^{-2}$ of a ML.

Despite adsorption on a real semiconductor surprisingly again magnetic field independent nuclear spin relaxation rates still linear in temperature were observed, which are about a factor of two smaller than the ones observed for the uncovered surface (Fig. 2). They do not depend on magnetic field strength (Fig. 4) and on bulk doping. Surprisingly, for all three kinds of preparation they display the same linear temperature dependence with an inverse slope with temperature of about $T_{1} T \approx 1000 \mathrm{~s} \mathrm{~K}$.

In discussing these results one should keep in mind that at hydrogen saturation the "cold" and "hot" hydrogen covered surface are far from being as perfect as the "wet" chemically terminated one. The "cold" prepared one consists still of isolated adatoms upon the rest layer and small islands which are made up of the missing adatoms from the exposed regions of the rest layer [28]. Also, the "hot" hydrogen covered surface exhibits numerous point defects, the most frequently occurring one being adatom trihydrides and top-layer atoms with a missing hydrogen adatom (dangling bond) [30]. But the almost identical $T_{1}$ times found for these Si(111):H surfaces and in particular their agreement with the $T_{1}$ times found for the almost perfect "wet" chemical hydrogen terminated Si(111)- $(1 \times 1)$ :H surface forces an interpretation which considers the adsorbed Li itself as the source of the fluctuating electron spins (moments) rather than, as for the uncovered surface, the electronic structure of the surface itself.

To gain at least a qualitative picture we recall that in the $\mathrm{Si}$ bulk $\mathrm{Li}$ is a donor with an ionization energy of $33 \mathrm{meV}[33,34]$. Thus, the donor electron wave function is quite extended and its Bohr radius amounts to about $20 \AA$. Moreover, with the dopant concentrations of the samples in mind (in between $10^{15} / \mathrm{cm}^{3}$ and $10^{18} / \mathrm{cm}^{3}$ ), the screening length [35] is always larger than $100 \AA$. Lacking other information we now assume that the radius is also typical of the extension of the Li donor on the hydrogen covered or terminated surface. Then at about $10^{-3} \mathrm{ML}$ the donor wave functions start to overlap, forming a band which determines the Fermi energy. Parallel, the Fermi level moves to the bottom of the conduction band. This picture is in accordance with high resolution electron energy loss spectroscopy (HREELS) experiments, which find at very low potassium coverage the formation of a charge accumulation layer (two-dimensional electron gas) at the surface of a hydrogenated $\mathrm{Si}(111)-(1 \times 1)$ crystal [36]. It is further corroborated by very recent density functional calculations [37]. 


\section{Adsorption of $\mathrm{Li}$ on a metal $(\operatorname{Ru}(001))$ surface}

The question of the "nature" of the alkali-metal bond towards a metal substrate is still debated. For metallic nearly free electrons, describable by the Bloch functions, the correlation time $\tau_{\mathrm{c}}$ approaches

$$
\tau_{\mathrm{c}}=\hbar \operatorname{DOS}\left(\epsilon_{\mathrm{F}}\right)
$$

and Eq. (3) takes the familiar form for "Korringa relaxation" in metals [14-16]. The local density of states at the nucleus and at the Fermi energy, defined as

$$
\operatorname{LDOS}\left(\epsilon_{\mathrm{F}}\right)=\left|\left\langle|\Psi(0)|^{2}\right\rangle\right|_{\mathrm{F}} \operatorname{DOS}\left(\epsilon_{\mathrm{F}}\right)
$$

is then the appropriate quantity to investigate the nature of the bond $[19,23]$. At an allotted temperature it can be determined directly from the relaxation rates if diffusional relaxation is suppressed sufficiently by high enough magnetic fields (here $0.35 \mathrm{~T}$ ) and low enough temperatures (here $100 \mathrm{~K}$ ). Careful checks showed that this choice satisfied the requirements for the experimental data to be discussed now.

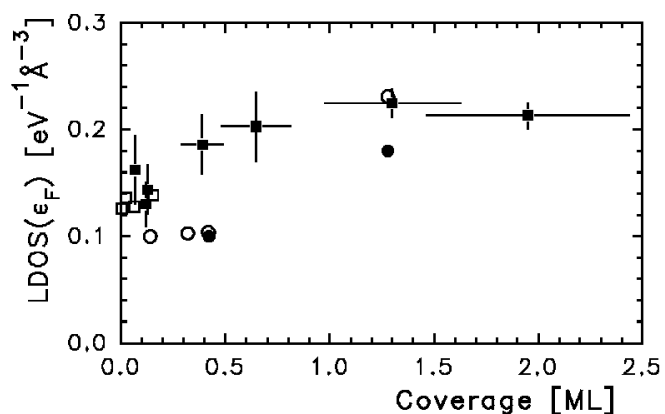

Fig. 5. For Li adsorption on a Ru(001) surface the local density of states at the nucleus and at the Fermi energy $\operatorname{LDOS}\left(\epsilon_{\mathrm{F}}\right)$ as a function of coverage. The open and full squares are experimental results, while the open and full round symbols indicate theoretical predictions.

Figure 5 displays the coverage dependence of $\operatorname{LDOS}\left(\epsilon_{\mathrm{F}}\right)$ determined from $T_{1}$ times through Eqs. (3), (5), and (6) (full squares) [38]. With respect to the number of substrate atoms of the surface 1 monolayer $(\mathrm{ML})$ corresponds to a coverage of 0.78 [39]. The open squares stem from previous measurements at $800 \mathrm{~K}$ and lower magnetic fields [23]. They are corrected for relaxation due to diffusion [23] and then according to $\mathrm{Eq}$. (3) rescaled to a temperature of $100 \mathrm{~K}$. The constant $\operatorname{LDOS}\left(\epsilon_{\mathrm{F}}\right)$ at low coverages and the associated linear decrease in the change of work function (not shown) was already understood as a result of a constant electric dipole moment per alkali-metal atom and the fact that the dipoles of the adsorbed Li atoms repel each other [23]. Since local electronic distortions are shielded quickly on metal surfaces, at low enough coverage all neighboring Li atoms experience the same electronic environment. 
Included into Fig. 5 are the predictions of all electron calculations of Mannstadt and Freeman (open dots) [40] together with the ones from our own group (full dots) [41]. Despite the value at highest coverage, both calculations agree quite well with each other, but disagree with the experiment in the medium coverage regime. They both underestimate $\operatorname{LDOS}\left(\epsilon_{\mathrm{F}}\right)$ slightly at low coverage. These all electron calculations deliver electron distributions which show an accumulation of electron density between the first Ru layer and the Li adsorbate, but certainly no indication, whatsoever, for an ionic bond.

\section{Summary and future prospects}

Obviously, NMR on single crystal surfaces is feasible using hyperpolarized species to enhance the sensitivity (up to now only alkali-metal atoms). The $\beta$-NMR experiments discussed need as little as $10^{4}$ equivalent nuclear spins per $\mathrm{cm}^{2}$ surface area. It is an enhancement of about 13 orders of magnitude as compared to the conventional technique. This success lives from two sources: on the one hand, through the high polarization of the adsorbed ${ }^{8} \mathrm{Li}$ a factor of about $10^{5}$ is gained as compared to conventional NMR experiments for which at moderate conditions ( $T=300 \mathrm{~K}, B=2 \mathrm{~T}$ ) the polarization amounts to about $10^{-5}$ only. On the other hand, one gains a factor of about $10^{8}$ through the "noiseless" counting of the $\beta$-decay electrons. About four orders of magnitude less gain in sensitivity is obtained in the experiments with the stable $\mathrm{Li}$ and $\mathrm{Na}$ isotopes $[2,3]$, since the detection of the nuclear polarization via laser induced fluorescence beam foil spectroscopy is by a factor of about $10^{4}$ less sensitive as compared to the $\beta$-electron detection.

As in real life, however, one has to pay a price: we need machines which are for a "normal" surface scientist quite exotic: accelerators, polarized sources, nuclear electronics, etc.. This and the fact that alkali-metal atoms are chemically also quite reactive probes limit this new method considerably. We have therefore looked carefully for alternatives and found that the noble gas Xe seems to be a good choice for an inert probe to study not only Xe adsorption but also adsorption of other species by Xe coadsorption. On the one hand, it can be highly polarized by polarization transfer from optically pumped $\mathrm{Rb}[42,43]$. Thus, with a signal enhancement of about five orders of magnitude even conventional NMR signal detection with an rf coil is feasible. This has been demonstrated with submonolayer amounts of Xe adsorbed on a liquid nitrogen cooled glass finger [44]. On the other hand, $\mathrm{Xe}$ is very sensitive to the physical and chemical properties of its local environment because of its high electric polarizability. This high sensitivity has already been used in NMR studies of large surface area materials [45, 46] and in a few cases even to study wall interactions already by the use of hyperpolarized ${ }^{129} \mathrm{Xe}$ or ${ }^{131} \mathrm{Xe}[47,48]$. The high electric polarizability also plays an important role for another single crystal surface analysis technique called photoemission spectroscopy 
of adsorbed Xe (PAX), which is used to sense the local potential of adsorption sites [49]. In addition to this, in the future spin transfer techniques might be able to study a large variety of other coadsorbed species containing ${ }^{13} \mathrm{C},{ }^{14} \mathrm{C},{ }^{29} \mathrm{Si}$, etc. directly $[50,51]$.

\section{References}

[1] C.P. Slichter, Ann. Rev. Phys. Chem. 37, 25 (1986).

[2] D. Fick, Appl. Phys. A 49, 343 (1989).

[3] H.J. Jänsch, Appl. Phys. A 65, 567 (1997).

[4] D. Fick, G. Grawert, I.M. Turkiewicz, Phys. Rep. 214, 1 (1992).

[5] M. Kaack, D. Fick, Phys. Rev. B 51, 17902 (1995).

[6] M. Eckhardt, H. Kleine, D. Fick, Surf. Sci. 319, 219 (1994).

[7] H.J. Jänsch, G. Kirchner, O. Kühlert, M. Lisowski, J.J. Paggel, R. Platzer, R. Schillinger, H. Tilsner, C. Weindel, H. Winnefeld, D. Fick, Nucl. Instrum. Methods Phys. Res. B 171, 537 (2000).

[8] H. Ackermann, P. Heitjans, H.-J. Stöckmann, in: Hyperfine Interactions of Radioactive Nuclei, Ed. J. Christiansen, Springer, Berlin 1983, p. 291.

[9] B. Ittermann, H. Ackermann, E. Diehl, B. Fischer, H.-P. Frank, H.-J. Stöckmann, Hyperfine Interact. 79, 591 (1993).

[10] W. Widdra, M. Detje, H.D. Ebinger, H.J. Jänsch, W. Preyß, H. Reich, R. Veith, D. Fick, M. Röckelein, H.-G. Völk, Rev. Sci. Instrum. 66, 2465 (1995).

[11] W. Preyß, H.D. Ebinger, H.J. Jänsch, R. Veith, D. Fick, M. Detje, C. Polenz, B. Polivka, Hyperfine Interact. 110, 295 (1997).

[12] M. Detje, M. Röckelein, W. Preyß, H.D. Ebinger, H.J. Jänsch, H. Reich, R. Veith, W. Widdra, D. Fick, J. Vac. Sci. Technol. A 13, 2532 (1995).

[13] H. Winnefeld, Ph.D. thesis, Philipps-Universität, Marburg 2000.

[14] A. Abragam, Principles of Nuclear Magnetism, University Press, Oxford 1978.

[15] G. Schatz, A. Weidinger, Nuclear Condensed Matter Physics, Wiley, Chichester 1996.

[16] C.P. Slichter, Principles of Magnetic Resonance, Springer, Berlin 1996.

[17] R. Losio, K.N. Altmann, F.J. Himpsel, Phys. Rev. B 61, 10845 (2000).

[18] D. Fick, R. Veith, H.D. Ebinger, H.J. Jänsch, C. Weindel, H. Winnefeld, J.J. Paggel, Phys. Rev. B 60, 8783 (1999).

[19] W. Mannstadt, G. Grawert, Phys. Rev. B 52, 5343 (1995).

[20] F. Flores, A.L. Yeyati, J. Ortega, Surf. Sci. Rev. Lett. 4, 281 (1997).

[21] J. Ortega, F. Flores, A.L. Yeyati, Phys. Rev. B 58, 4584 (1998).

[22] K.D. Brommer, M. Galván, J.A. Dal Pino, J.D. Joannopoulos, Surf. Sci. 314, 57 (1994).

[23] H.D. Ebinger, H. Arnolds, C. Polenz, B. Polivka, W. Preyß, R. Veith, D. Fick, H.J. Jänsch, Surf. Sci. 412/413, 586 (1998).

[24] W.W. Warren, Jr., Phys. Rev. B 3, 3708 (1971). 
[25] H.H. Weitering, J. Chen, N.J. DiNardo, E.W. Plummer, Phys. Rev. B 48, 8119 (1993).

[26] C. Weindel, Ph.D. thesis, Philipps-Universität, Marburg 2000.

[27] A. Körblein, P. Heitjans, H.-J. Stöckmann, F. Fujara, H. Ackermann, W. Buttler, K. Dörr, H. Grupp, J. Phys. F 15, 561 (1985).

[28] J. Boland, Surf. Sci. 244, 1 (1991).

[29] K. Mortensen, D.M. Chen, P.J. Bedrossian, J.A. Golovchenko, F. Besenbacher, Phys. Rev. B 43, 1816 (1991).

[30] F. Owman, P. Martensson, Surf. Sci. 324, 211 (1995).

[31] Y. Morita, K. Miki, H. Tokumoto, Surf. Sci. 325, 21 (1995).

[32] Y.J. Chabal, J.E. Rowe, D.A. Zwemer, Phys. Rev. Lett. 46, 600 (1981).

[33] C.S. Fuller, J.A. Ditzenberger, Phys. Rev. 91, 193 (1953).

[34] G. Feher, Phys. Rev. 114, 1219 (1959).

[35] J.M. Ziman, Principles of the Theory of Solids, University Press, Cambridge 1964.

[36] R. Biagi, L. Rettighieri, U. del Pennino, V. Panella, P. Dumas, Surf. Sci. 402, 547 (1998).

[37] W. Mannstadt, private communication, 2001.

[38] G. Kirchner, Ph.D. thesis, Philipps-Universität, Marburg 1999.

[39] M. Gierer, H. Over, H. Bludau, G. Ertl, Phys. Rev. B 52, 2927 (1995).

[40] W. Mannstadt, A.J. Freeman, Phys. Rev. B 57, 13289 (1998).

[41] L. Hufnagel, diploma thesis, Philipps-Universität, Marburg 1998.

[42] T.G. Walker, W. Happer, Rev. Mod. Phys. 69, 629 (1997).

[43] U. Ruth, T. Hof, J. Schmidt, D. Fick, H.J. Jänsch, Appl. Phys. B 68, 93 (1999).

[44] H.J. Jänsch, T. Hof, U. Ruth, J. Schmidt, D. Stahl, D. Fick, Chem. Phys. Lett. 296, 146 (1998).

[45] M. Tomaselli, B.H. Meier, P. Rogyr, U.W. Suter, R.R. Ernst, Chem. Phys. Lett. 205, 145 (1993).

[46] A. Bifone, T. Pietrass, J. Kritzenberger, A. Pines, B.F. Chmelka, Phys. Rev. Lett. 74, 3277 (1995).

[47] R. Butscher, G. Wäckerle, M. Mehring, J. Chem. Phys. 100, 6923 (1994).

[48] D. Raftery, L. Reven, H. Long, A. Pines, P. Tang, J.A. Reimer, J. Phys. Chem. 97, 1649 (1993).

[49] K. Wandelt, in: Thin Metal Films and Gas Chemisorption, Ed. P. Wissmann, Vol. 32 of Studies in Surface Science and Catalysis, Elsevier, Amsterdam 1987, p. 280.

[50] T. Room, S. Appelt, R. Seydoux, E.L. Hahn, A. Pines, Phys. Rev. B 55, 11604 (1997).

[51] H.W. Long, H.C. Gaede, J. Shore, L. Reven, C.R. Bowers, J. Kritzenberger, T. Pietrass, A. Pines, P. Tang, J.A. Reimer, J. Am. Chem. Soc. 115, 8491 (1993). 